\title{
Mechanisms, impact and prevention of pathological bone regeneration in spondyloarthritis
}

Barbara Neerinckx and Rik Lories

Laboratory of Tissue Homeostasis and Disease, Skeletal Biology and Engineering Research Center, Department of Development and Regeneration, KU Leuven \& Division of Rheumatology, University Hospitals Leuven.

Corresponding author: Prof. R. Lories, Division of Rheumatology, UZ Leuven, Herestraat 49, 3000 Leuven, Belgium. Phone: +32-16-342541; Fax: +32-16-342543; Rik.Lories@uzleuven.be

\begin{abstract}
Purpose of the review: to discuss different aspects of new bone formation in patients with spondyloarthritis based on emerging data from clinical trials, prospective cohort studies and translational laboratory investigations.

Recent findings: new bone formation potentially leading to ankylosis of the spine and sacroiliac joints remains an important concern for patients with axial spondyloarthritis. New therapeutic strategies, in particular targeting of interleukin17 have emerged in addition to the anti-tumor necrosis factor drugs but we still fail to fully understand the mechanisms of structural disease progression. A new paradigm is developing in which sustained and effective suppression of inflammation likely inhibits this structural disease progression. Biomechanical factors, in particular changes in bone microarchitecture in the vertebrae, and the need for core stability could provide a new framework to understand the relationship between bone remodeling and inflammation and to develop long-term strategies.

Summary: New bone formation leading to ankylosis remains a hallmark of axial spondyloarthritis and should be further investigated. The clinical data that progressively become available support the concept that effective and sustained therapy will be beneficial for the patients not only in short-term but also in longterm outcomes.
\end{abstract}

Keywords: spondyloarthritis, bone, ankylosis, ankylosing spondylitis

Disclosure of funding: B.N. was the recipient of an "Aspirant" fellowship from the Flanders Research Foundation (FWO Vlaanderen). Original research by the authors was supported by grant G.0946.14 from FWO Vlaanderen and an "OT"-grant from KU Leuven. 



\section{Introduction}

Spondyloarthritis is a chronic inflammatory skeletal disease that covers different diagnostic entities that share clinical, genetic, pathophysiological and radiographic characteristics [1]. Currently, the location of the main clinical presentations helps to distinguish axial spondyloarthritis, including ankylosing spondylitis and nonradiographic axial spondyloarthritis, and peripheral spondyloarthritis. In axial spondyloarthritis the dominant symptom is inflammatory back pain and stiffness. Peripheral spondyloarthritis covers more heterogeneous presentations. Many patients in this group are diagnosed with psoriatic arthritis and with inflammatory bowel disease associated arthritis. Here, oligoarthritis of the lower limbs and clinical enthesitis, for example at the Achilles tendon insertion or the plantar fascia, are key manifestations. However, polyarticular disease involving small joints of the hand is not uncommon, in particular in the group of patients with psoriatic arthritis.

Structural damage to the skeleton as a consequence of chronic arthritis is clinically important as it contributes strongly to loss of function and disability. In axial spondyloarthritis the sacroiliac joints and the spine are typically sites where such damage occurs. Progressive disease potentially translates into bridging of the sacroiliac and the facet joints and the formation of bridging syndesmophytes between vertebral bodies. Bone destructive lesions also occur, in particular erosion of the sacroiliac joints or end-plates of the vertebra but appear to represent a more transient phase followed by the bone remodeling process that leads to ankylosis. Equally important from a clinical and from a pathophysiological perspective is the inflammation-associated bone loss that occurs in the spine, increasing strongly the risk for fractures in patients with axial spondyloarthritis. As further elaborated below, this bone loss and the associated changes in the bone microarchitecture have been proposed as a potential driving mechanism for the ankylosing process [2]. In peripheral joints both bone erosion and bone remodeling can be found. Many affected joints show varying degrees of new bone formation but the development of full joint ankylosis is rare, in particular in the larger joints.

The management of spondyloarthritis and the prospects for the individual patients have dramatically changed after the turn of the century. The introduction of targeted strategies that aim to neutralize the cytokine tumor necrosis factor (TNF) has lead to unprecedented control of inflammation in a large number of patients [1]. More recently a second wave of drug development seems to finds its way into clinical practice. A novel strategy directed at neutralization of pro-inflammatory interleukin-17 (IL17) spearheads the arrival of new treatments [3, 4]. In addition to market approved anti-IL17 there are also early data available and clinical trials underway evaluating the effect of anti-IL12/23 [5] as well as studying small 
molecules such as JAK/STAT inhibitor tofacitinib [6] or phosphodiesterase 4 inhibitor apremilast [7].

Whereas the clinical effect of anti-TNF strategies has been undisputable for a large number of patients, the effect of such interventions on structural disease progression is less clear $[8,9]$. In particular the early post-clinical trial cohort studies comparing long-term follow-up of anti-TNF treatment with a historical cohort casted doubts about a structure modifying effect on these drugs [10]. However, novel data from studies with TNF inhibitors and with the new drugs available are slowly bringing evidence that prevention of structural damage may not be an unachievable goal anymore.

\section{The importance and impact of progressive structural damage in axial spondyloarthritis}

Individual variation in the extent, speed of progression and impact of structural damage, in particular in the axial forms, is highly variable [11]. Nevertheless it is an important concern and point of attention for many patients and physicians for various reasons. The distinction between ankylosing spondylitis and the nonradiographic forms is largely made by the presence of specific changes in the radiographic evaluation of the sacroiliac joints. The newly developed classification criteria for axial spondyloarthritis [12] and the delineation of non-radiographic axial spondyloarthritis as a specific entity have stirred up some controversy [13, 14]. From a practical and patient-focused point of view the distinction between ankylosing spondylitis and non-radiographic axial spondyloarthritis should be best abandoned in diagnosis and management of disease and only used for classification in clinical and translational studies [13]. The debate did identify the important remaining concern that we do not sufficiently understand the time-course of disease in individual patients and in particular ignore the answer to the question which patients will ultimately develop ankylosis.

Progressive ankylosis strongly contributes to the burden of the disease and resulting disability. Older data presented by Machado et al. clearly identified that both inflammation and structural damage define the effect of axial spondyloarthritis on the patient [15]. Inflammation appears to be most important in the early disease phase whereas structural damage takes steps to the foreground as disease duration increases. Over the last couple of years further insights have been provided into specific features of the disease and their impact on the patient. Poddubnyy et al., studied a cohort of patients from open-label extensions of clinical trials testing the effects of either infliximab or etanercept in patients with ankylosing spondylitis. Despite the observation that this cohort of successfully treated patients did show 
radiographic progression, their functional status and the assessment of the spinal mobility remained stable over time [16]. This supports the view that sustained control of inflammation results in disease modification at the clinical level even if the definition of structural modification at the radiographic level is not met [17].

\section{Is treatment of inflammation capable of preventing structural disease progression?}

The great clinical success associated with the introduction of different TNF inhibitors in clinical practice has sparked expectations that structural damage, and in particular new bone formation leading to ankylosis in the spine, would also be prevented by the successful intervention. This initial expectation resulted in disappointment. Twoyear follow up studies with different tumor necrosis factor antagonists did not find a difference in radiographic progression compared to the historical prospective OASIS cohort [18-20]. However, more recent data strongly suggest that sustained TNF inhibition over a much longer time period may result in effective inhibition of radiographic progression. Baraliakos et al., reported on a cohort of ankylosing spondylitis patients treated with infliximab [21]. Although by the end of their prospective evaluation, the sample number of patients became low, curves between treated and non-treated patients became clearly different. Haroon et al., also suggested that anti-TNF treatments in particular when started early would have an impact on radiographic progression [22]. More recently Maas et al. also reported their prospective cohort follow-up with patients being exposed to anti-TNF treatment for up to eight years. Remarkably, structural disease progression continued in a linear way up to 4 years after the initiation of anti-TNF therapy. However, in the next phase the measured disease progression was strongly reduced and interrupted the linear course [23]. Taken together these novel data could provide support for an emerging view that we proposed earlier in this journal on structural disease progression in patients with axial spondyloarthritis [2] (Figure 1).

Inflammation at the spine leads to trabecular bone loss and increased fracture risk, in particular when there is osteitis in the vertebral bodies [24, 25]. Inflammation therefore likely affects the bone's microarchitecture. Loss of this bone microarchitecture in a part of the body where dynamic loading is important is hypothesized to trigger a regenerative or reparative response [2]. Since restoring bone quality in the trabecular bone may be difficult if inflammation persists, stabilizing new bone formation occurs at the vertebral corners and the facet joints. In this hypothesis, progressive ankylosis is mainly driven by bone instability and will improve after sustained treatment of inflammation. This would also imply that, in addition to continued and efficacious treatment to control inflammation, dedicated physiotherapy aiming at increasing core stability and proprioception are important factors for intervention [2]. The importance of rapid stabilization as driving force for 
ankylosis appears to be confirmed in another recent study by Maas et al. Here, the authors show that structural disease progression in the cervical spine even under anti-TNF therapy, rather involved the cervical facet joints that the vertebral bodies [26].

Among the new strategies, secukinumab, a monoclonal antibody directed against interleukin-17A, is currently used in the clinic for the treatment of axial spondyloarthritis [3, 4], psoriasis [27] and psoriatic arthritis [28]. The clinical effect size at the group level of secukinumab treatment appears comparable to that of TNF inhibitors and the drug appears to be effective in a significant proportion of patients that failed anti-TNF therapy [3, 4]. A first analysis after two years did not document significant structural disease progression in axial spondyloarthritis [29]; however there are different caveats that should be considered before claiming that anti-IL17A has stronger effects that TNF on structural disease progression. Although classical parameter comparisons do not suggest strong differences in selected patient populations, patients with spondyloarthritis are likely better diagnosed and treated in this decennium that in the previous one. Patients with high risk of radiographic progression may be treated earlier and better than before. Thus, it is unclear if the observed data with anti-17 represent an effect of shifts in the patient population included in trials or a drug effect. Additional data will be necessary to fully understand the impact of novel drugs on radiographic progression.

Data on non-steroidal anti-inflammatory drug (NSAID) use in patients with axial spondyloarthritis, still the first line treatment, also remain somewhat controversial. Continuous NSAID intake could have a significant effect on structural disease progression [30, 31]. However, The ENRADA trial did not confirm this observation [32]. Here, radiographic progression was not statistically different between patients receiving continuous or on-demand diclophenac. Again, differences in patient population included in clinical trials from different decades may explain this inconsistency. Patients with severe inflammation and high risk for radiographic progression may more rapidly be considered for advanced treatments. A post-study analysis of the trial from Wanders et al. [30] indeed indicated that the patient population that benefited most from continuous NSAID treatment were those patients with elevated CRP probably reflecting severe disease [33].

\section{Inflammation and new bone formation, still a difficult question?}

The relationship between inflammation and new bone formation has been strongly debated since the first data on radiographic progression in patients treated with anti-TNF became available $[8,10]$. Increasing evidence supports the view that inflammation and ankylosis are linked but molecularly and cellular uncoupled 
processes $[8,34,35]$. Many aspects of this view are based on the sequential analysis of disease sites by nuclear magnetic resonance imaging (MRI) [36, 37]. Fatty lesions have been proposed as a transitional phase between active inflammation and new bone formation [35, 38] [39], and the tissue metaplasia seen in these lesions has recently been confirmed by histology [40].

The molecular pathways that stimulate new bone formation appear clearly distinct from the classical inflammatory cascades that are associated with the disease and that are currently targeted by available and upcoming drugs. When discussing new bone formation, growth factor signaling cascades such as bone morphogenetic proteins (BMPs), Wnts and Hedgehogs come into the focus [8]. Inhibition of Dickkopf-1 (DKK1), an antagonist of the Wnt signaling pathway, shifts erosive arthritis in mice towards ankylosing disease [41]. Inhibition of BMP signaling by overexpression of its antagonist noggin was earlier shown to protect against the development of joint ankylosis in mice [42]. Hedgehog signaling has been specifically associated with chondrocyte hypertrophy [43]. A chemical antagonist of the pathway inhibits new bone formation in different mouse models [44].

The impact of inflammatory cytokines on these processes remains largely unclear. Sherlock et al., suggested that IL-22 production triggered by systemic overexpression of IL-23 is a key player in new bone formation [45] but further evidence for this hypothesis has only come from specific in vitro experiments that lack further in vivo validation [46]. Other in vitro experiments have further investigated the link between inflammatory cytokines, growth factor cascades and cell differentiation assays. TNF and IL-17 have a mostly negative impact on setups focused on chondroor osteogenic differentiation, an in vitro observation that appears to be confirmed in vivo. High levels of TNF such as seen in the human TNF transgenic mouse model, appear to complete inhibit bone remodeling and repair [41]. Nevertheless, in some experimental setups negative effects of IL17 and TNF on the bone forming process appear to be inversed. The differentiation status of the cells used in such setups appears to be a key determinant of the outcome. For instance in cells that have already committed to bone formation IL-17 and BMP2 appear to have remarkable synergistic effects in stimulating the process [47].

\section{Monitoring of bone remodeling in spondyloarthritis}

Biomarker studies are facing many challenges in this context. Many of the available reports are based on cross-sectional sampling and do not provide sufficient followup to allow a full prospective view on the data. There is an unmet need for large prospective evaluations that set high standards for sample taking, its timing and storage. Any evaluation of markers associated with bone remodeling needs to take 
into consideration that effects on bone at the systemic level, associated with inflammation, can strongly impact the potential to measure events at a smaller scale, for instance in the developing syndesmophyte. There is a need for combined and innovative biomarker and imaging studies as growth factor concentrations at the local level are much more relevant than their systemic presence. Indeed, molecules associated with embryonic skeletal development such as BMP and Wnts exert there effects by local concentration gradients established in complex connective tissues that are rich in extracellular matrix molecules, thus representing an enormous challenge for systemic sampling and analysis.

Some data are available however and suggest that further investment in these questions may be warranted. DKK1 and Wnt signaling have been best studied. The original report on DKK1 from Diarra et al. indicated that spondyloarthritis patients would have very low levels of DKK1 [41]. Additional data demonstrated that high levels of functional DKK1 predict protection against radiographic progression [48]. However, Daoussis et al., intriguingly showed that levels of DKK1 are elevated in patients with axial spondyloarthritis but that DKK1 itself is not functional [49]. Indeed functional DKK-1 levels appear to be lower in the serum of patients with ankylosing spondylitis compared to controls, and levels are inversely correlated with progression of ankylosis [50]. Such data were also reported for sclerostin, another Wnt antagonist $[48,51]$. Data on BMPs are less convincing and consistent, mostly due to the small cohort sizes.

\section{Conclusion}

The opportunities to effectively treat patients with spondyloarthritis are still growing and control of inflammation and associated symptoms is an increasingly achievable goal. Nevertheless progressive ankylosis remains a concern. As patients are treated earlier and more consistently these therapies also appear to have an impact on structural disease progression. Inflammation resulting in bone loss may be the driving factor of bone remodeling in this disease. Further research is still required in particular to better understand the relationship between the key inflammatory cytokines that play a role in spondyloarthritis and the growth factors that steer bone remodeling. From a clinical perspective, carefully designed prospective cohorts will be necessary to confirm the evolving hypotheses.

\section{Key points}

1. Long-term follow up data of patients with spondyloarthritis treated with antitumor necrosis factor drug indicate slow but successful effects of the durgs on ankylosis. 
2. Loss of bone with changes in micro-architecture could be the drivers of the ankylosing process.

3. Early data with interleukin-17 blocker secukinumab warrant optimism for effects on radiographic progression in axial spondyloarthritis.

4. Further research on the relationship between inflammatory cytokines, growth factors and target cells is required to move the field forward.

\section{Acknowledgements and Conflicts of Interest}

B.N. was the recipient of an "Aspirant" fellowship from the Flanders Research Foundation (FWO Vlaanderen). Original research by the authors was supported by grant G.0946.14 from FWO Vlaanderen and an "OT"-grant from KU Leuven. Leuven Research and Development, the technology transfer office of KU Leuven has received speaker's and consultancy fees on behalf of R.L from Abbvie, BoehringerIngelheim, Celgene, Janssen, Novartis, Merck and Pfizer.

\section{Figure 1}

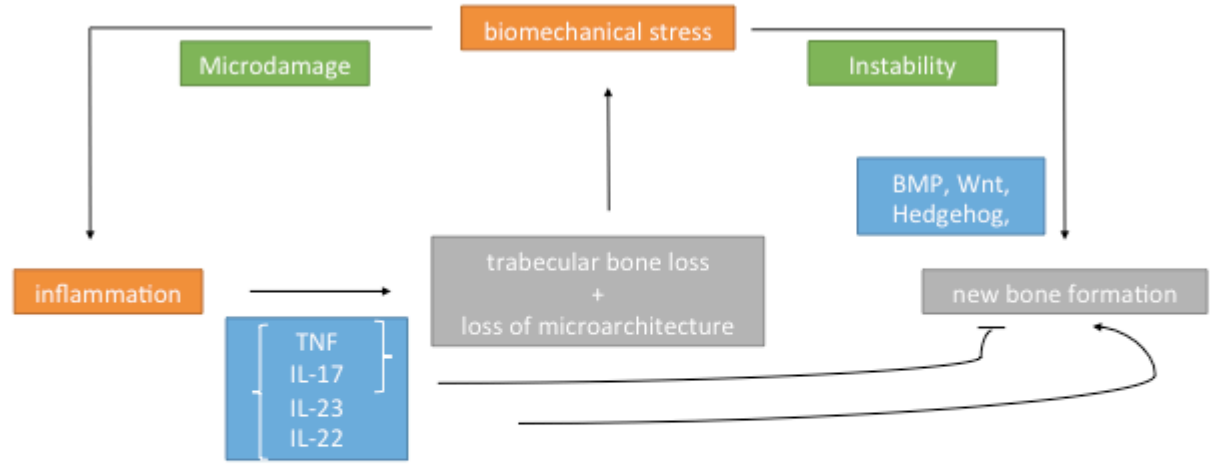

Figure 1: Impact of biomechanical factors in spondyloarthritis. Biomechanical stress can contribute to inflammation by causing microdamage. Cytokines produced can lead to bone loss and instability resulting in a different type of biomechanical stress that triggers new bone formation to stabilize the spine or joint. Cytokines like TNF and IL-17 negatively affect bone formation in vitro, whereas IL-22 can stimulate bone formation. Bone morphogenetic proteins (BMPs), Wnts and Hedgehog $(\mathrm{HH})$ proteins directly stimulate ankylosis. 


\section{References}

1. Dougados M, Baeten D. Spondyloarthritis. Lancet. 2011;377(9783):2127-37.

* 2. Van Mechelen M, Lories RJ. Microtrauma: no longer to be ignored in spondyloarthritis? Curr Opin Rheumatol. 2016;28(2):176-80.

In this paper, the concept of bone loss driving ankylosis is extensively discussed.

3. Baeten D, Baraliakos X, Braun J et al. Anti-interleukin-17A monoclonal antibody secukinumab in treatment of ankylosing spondylitis: a randomised, double-blind, placebo-controlled trial. Lancet. 2013;382(9906):1705-13.

* 4. Baeten D, Sieper J, Braun J et al. Secukinumab, an Interleukin-17A Inhibitor, in Ankylosing Spondylitis. N Engl J Med. 2015;373(26):2534-48.

Pivotal trial showing the strong effect of anti-IL17A on clinical disease manifestations in patients with ankylosing spondylitis.

5. Poddubnyy D, Hermann KG, Callhoff J et al. Ustekinumab for the treatment of patients with active ankylosing spondylitis: results of a 28-week, prospective, openlabel, proof-of-concept study (TOPAS). Ann Rheum Dis. 2014;73(5):817-23.

* 6. van der Heijde D, Deodhar A, Wei JC et al. Tofacitinib in patients with ankylosing spondylitis: a phase II, 16-week, randomised, placebo-controlled, dose-ranging study. Ann Rheum Dis. 2017. E-pub

Early data on the effect of JAK/STAT inhibition in patients with ankylosing spondylitis.

7. Pathan E, Abraham S, Van Rossen E et al. Efficacy and safety of apremilast, an oral phosphodiesterase 4 inhibitor, in ankylosing spondylitis. Ann Rheum Dis. 2013;72(9):1475-80.

8. Lories RJ, Schett G. Pathophysiology of new bone formation and ankylosis in spondyloarthritis. Rheum Dis Clin North Am. 2012;38(3):555-67.

9. Lories RJ, Haroon N. Bone formation in axial spondyloarthritis. Best Pract Res Clin Rheumatol. 2014;28(5):765-77.

10. Maksymowych WP, Elewaut D, Schett G. Motion for debate: the development of ankylosis in ankylosing spondylitis is largely dependent on inflammation. Arthritis Rheum. 2012;64(6):1713-9. 
11. Baraliakos X, Listing J, von der Recke A et al. The natural course of radiographic progression in ankylosing spondylitis--evidence for major individual variations in a large proportion of patients. J Rheumatol. 2009;36(5):997-1002.

12. Rudwaleit $M$, van der Heijde D, Landewe R et al. The development of Assessment of SpondyloArthritis international Society classification criteria for axial spondyloarthritis (part II): validation and final selection. Ann Rheum Dis. 2009;68(6):777-83.

** 13. Deodhar A, Strand V, Kay J et al. The term 'non-radiographic axial spondyloarthritis' is much more important to classify than to diagnose patients with axial spondyloarthritis. Ann Rheum Dis. 2016;75(5):791-4.

Important and thougtful considerations about the dangers associated with the use of classification criteria in daily clinical practice.

14. van der Linden $S$, Akkoc N, Brown MA et al. The ASAS Criteria for Axial Spondyloarthritis: Strengths, Weaknesses, and Proposals for a Way Forward. Curr Rheumatol Rep. 2015;17(9):62.

15. Machado $P$, Landewe $R$, Braun J et al. Both structural damage and inflammation of the spine contribute to impairment of spinal mobility in patients with ankylosing spondylitis. Ann Rheum Dis. 2010;69(8):1465-70.

** 16. Poddubnyy D, Fedorova A, Listing J et al. Physical Function and Spinal Mobility Remain Stable Despite Radiographic Spinal Progression in Patients with Ankylosing Spondylitis Treated with TNF-alpha Inhibitors for Up to 10 Years. J Rheumatol. 2016;43(12):2142-8.

This paper provides a clear confirmation of the concept that disease modification entails more than radiographic progression alone.

17. Lories RJ, de Vlam K, Luyten FP. Are current available therapies diseasemodifying in spondyloarthritis? Best Pract Res Clin Rheumatol. 2010;24(5):625-35.

18. van der Heijde $D$, Landewe $R$, Baraliakos $X$ et al. Radiographic findings following two years of infliximab therapy in patients with ankylosing spondylitis. Arthritis Rheum. 2008;58(10):3063-70.

19. van der Heijde $D$, Landewe $R$, Einstein $S$ et al. Radiographic progression of ankylosing spondylitis after up to two years of treatment with etanercept. Arthritis Rheum. 2008;58(5):1324-31. 
20. van der Heijde D, Salonen D, Weissman BN et al. Assessment of radiographic progression in the spines of patients with ankylosing spondylitis treated with adalimumab for up to 2 years. Arthritis Res Ther. 2009;11(4):R127.

21. Baraliakos $\mathrm{X}$, Haibel $\mathrm{H}$, Listing J et al. Continuous long-term anti-TNF therapy does not lead to an increase in the rate of new bone formation over 8 years in patients with ankylosing spondylitis. Ann Rheum Dis. 2014;73(4):710-5.

22. Haroon N, Inman RD, Learch TJ et al. The impact of tumor necrosis factor alpha inhibitors on radiographic progression in ankylosing spondylitis. Arthritis Rheum. 2013;65(10):2645-54.

* 23. Maas F, Arends S, Brouwer E et al. Reduction in spinal radiographic progression in ankylosing spondylitis patients receiving prolonged treatment with TNF-alpha inhibitors. Arthritis Care Res (Hoboken). 2016. E-pub.

Further confirmation that long-term treatment with TNF inhibitors affects radiographic progression of disease in axial spondyloarthritis.

24. Carter S, Lories RJ. Osteoporosis: a paradox in ankylosing spondylitis. Curr Osteoporos Rep. 2011;9(3):112-5.

* 25. Maas F, Spoorenberg A, van der Slik BP et al. Clinical risk factors for the presence and development of vertebral fractures in patients with ankylosing spondylitis. Arthritis Care Res (Hoboken). 2016. E-pub.

This papers highlights the bone loss seen in patients with ankylosing spondylitis.

** 26. Maas F, Spoorenberg A, Brouwer $E$ et al. Radiographic damage and progression of the cervical spine in ankylosing spondylitis patients treated with TNFalpha inhibitors: Facet joints vs. vertebral bodies. Semin Arthritis Rheum. 2016. Epub.

Interesting observation that ankylosis of the facet joints may be more important than vertebral bridging in the cervical spine.

27. Langley RG, Elewski BE, Lebwohl $M$ et al. Secukinumab in plaque psoriasis-results of two phase 3 trials. N Engl J Med. 2014;371(4):326-38.

28. McInnes IB, Sieper J, Braun J et al. Efficacy and safety of secukinumab, a fully human anti-interleukin-17A monoclonal antibody, in patients with moderate-tosevere psoriatic arthritis: a 24-week, randomised, double-blind, placebo-controlled, phase II proof-of-concept trial. Ann Rheum Dis. 2014;73(2):349-56. 
29. Baraliakos X, Deodhar AA, Braun J et al. Effect of Interleukin-17A Inhibition on Spinal Radiographic Changes through 2 Years in Patients with Active Ankylosing Spondylitis: Results of a Phase 3 Study with Secukinumab. Arthritis \& Rheumatology. 2015;67:3.

30. Wanders A, Heijde D, Landewe $R$ et al. Nonsteroidal antiinflammatory drugs reduce radiographic progression in patients with ankylosing spondylitis: a randomized clinical trial. Arthritis Rheum. 2005;52(6):1756-65.

31. Poddubnyy D, Rudwaleit $M$, Haibel $H$ et al. Effect of non-steroidal antiinflammatory drugs on radiographic spinal progression in patients with axial spondyloarthritis: results from the German Spondyloarthritis Inception Cohort. Ann Rheum Dis. 2012;71(10):1616-22.

** 32. Sieper J, Listing J, Poddubnyy D et al. Effect of continuous versus on-demand treatment of ankylosing spondylitis with diclofenac over 2 years on radiographic progression of the spine: results from a randomised multicentre trial (ENRADAS). Ann Rheum Dis. 2016;75(8):1438-43.

Relatively large clinical trial challenging the earlier concept that NSAIDs will delay radiographic progression of disease in ankylosing spondylitis.

33. Kroon $\mathrm{F}$, Landewe $\mathrm{R}$, Dougados $\mathrm{M}$ et al. Continuous NSAID use reverts the effects of inflammation on radiographic progression in patients with ankylosing spondylitis. Ann Rheum Dis. 2012;71(10):1623-9.

34. Maksymowych WP. Disease modification in ankylosing spondylitis. Nature Reviews Rheumatology. 2010;6(2):75-81.

35. Maksymowych WP, Morency N, Conner-Spady B et al. Suppression of inflammation and effects on new bone formation in ankylosing spondylitis: evidence for a window of opportunity in disease modification. Annals of the Rheumatic Diseases. 2013;72(1):23-8.

36. Maksymowych WP, Wichuk S, Chiowchanwisawakit P et al. Fat Metaplasia and Backfill Are Key Intermediaries in the Development of Sacroiliac Joint Ankylosis in Patients With Ankylosing Spondylitis. Arthritis \& Rheumatology. 2014;66(11):295867.

37. Chiowchanwisawakit P, Lambert RGW, Conner-Spady B et al. Focal Fat Lesions at Vertebral Corners on Magnetic Resonance Imaging Predict the Development of New Syndesmophytes in Ankylosing Spondylitis. Arthritis Rheum-Us. 2011;63(8):2215-25.

* 38. Machado PM, Baraliakos X, van der Heijde D et al. MRI vertebral corner inflammation followed by fat deposition is the strongest contributor to the 
development of new bone at the same vertebral corner: a multilevel longitudinal analysis in patients with ankylosing spondylitis. Annals of the Rheumatic Diseases. 2016;75(8):1486-93.

Further insights into the sequence of changes seen by MRI during the development of syndesmophytes.

39. Baraliakos X, Heldmann F, Callhoff J et al. Which spinal lesions are associated with new bone formation in patients with ankylosing spondylitis treated with antiTNF agents? A long-term observational study using MRI and conventional radiography. Annals of the Rheumatic Diseases. 2014;73(10):1819-25.

** 40. Baraliakos X, Boehm H, Samir A et al. Which Cells Correspond to Typical Signals for Fatty and Inflammatory Lesions Seen on Mri in As? Clinical and Experimental Rheumatology. 2016;34(4):744.

Important study providing histology images of the fatty lesions earlier recognized by MRI imaging.

41. Diarra $D$, Stolina $M$, Polzer $K$ et al. Dickkopf-1 is a master regulator of joint remodeling. Nat Med. 2007;13(2):156-63.

42. Lories RJ, Derese I, Luyten FP. Modulation of bone morphogenetic protein signaling inhibits the onset and progression of ankylosing enthesitis. J Clin Invest. 2005;115(6):1571-9.

43. Lefebvre V, Bhattaram P. Vertebrate skeletogenesis. Curr Top Dev Biol. 2010;90:291-317.

44. Ruiz-Heiland G, Horn A, Zerr P et al. Blockade of the hedgehog pathway inhibits osteophyte formation in arthritis. Ann Rheum Dis. 2012;71(3):400-7.

45. Sherlock JP, Joyce-Shaikh B, Turner SP et al. IL-23 induces spondyloarthropathy by acting on ROR-gammat+ CD3+CD4-CD8- entheseal resident T cells. Nat Med. 2012;18(7):1069-76.

46. El-Sherbiny $Y$, Elzayadi A, Fragkakis EM et al. IL-22 Drives the Proliferation and Differentiation of Human Bone Marrow Mesenchymal Stem Cells (MSCs); A Novel Pathway That May Contribute to Aberrant New Bone Formation in Human Spa and Beyond. Arthritis \& Rheumatology. 2015;67.

** 47. Croes M, Oner FC, van Neerven D et al. Proinflammatory T cells and IL-17 stimulate osteoblast differentiation. Bone. 2016;84:262-70. 
Novel data demonstrating potential synergism between IL17 and growth factors that stimulate bone formation.

48. Heiland GR, Appel H, Poddubnyy D et al. High level of functional dickkopf-1 predicts protection from syndesmophyte formation in patients with ankylosing spondylitis. Annals of the Rheumatic Diseases. 2012;71(4):572-4.

49. Daoussis D, Liossis SN, Solomou EE et al. Evidence that Dkk-1 is dysfunctional in ankylosing spondylitis. Arthritis Rheum. 2010;62(1):150-8.

50. Yucong Z, Lu L, Shengfa L et al. Serum functional dickkopf-1 levels are inversely correlated with radiographic severity of ankylosing spondylitis. Clin Lab. 2014;60(9):1527-31.

51. Nocturne G, Pavy S, Boudaoud S et al. Increase in Dickkopf-1 Serum Level in Recent Spondyloarthritis. Data from the DESIR Cohort. PLoS One. 2015;10(8):e0134974. 